PAPER • OPEN ACCESS

Neutron star dynamics under time dependent external torques

To cite this article: M A Alpar and E Gügercinolu 2017 J. Phys.: Conf. Ser. 932012036
Related content

VORTEX CREEP AGAINST TOROIDAL AND PULSAR GLITCHES

Erbil Gügercinolu and M. Ali Alpar

Spin Evolution of Pulsars

Armen Sedrakian and James M. Cordes

- Torque measurement issues

J Goszczak

View the article online for updates and enhancements. 


\title{
Neutron star dynamics under time dependent external torques
}

\author{
M A Alpar and E Gügercinoğlu \\ Faculty of Engineering and Natural Sciences, Sabancı University, Orhanlı -Tuzla, İstanbul \\ 34956, Turkey \\ E-mail: alpar@sabanciuniv.edu
}

\begin{abstract}
The two component model of neutron star dynamics describing the behaviour of the observed crust coupled to the superfluid interior has so far been applied to radio pulsars for which the external torques are constant on dynamical timescales. We recently solved this problem under arbitrary time dependent external torques. Our solutions pertain to internal torques that are linear in the rotation rates, as well as to the extremely non-linear internal torques of the vortex creep model. Two-component models with linear or nonlinear internal torques can now be applied to magnetars and to neutron stars in binary systems, with strong variability and timing noise. Time dependent external torques can be obtained from the observed spin-down (or spin-up) time series, $\dot{\Omega}(t)$.
\end{abstract}

\section{The two component model with constant external torques}

The two component model for neutron star dynamics was proposed immediately after the observation of the first pulsar glitches [1]. This model and its extension to nonlinear vortex creep coupling between the crust and the superfluid [2] have been applied with remarkable success to the postglitch relaxation data from radio pulsars, for which the external torques are constant on dynamical timescales. This talk presents, in summary, our recent solution of the two component models for linear and nonlinear coupling for the general case of arbitrary time dependent external torques [3]. These general solutions are applicable to the timing behaviour of neutron stars in X-ray binaries and magnetars, as well as timing noise from radio pulsars.

Postglitch exponential relaxation on timescales of days to weeks supported the theoretical expectations of superfluidity in the neutron star interior. As exponential relaxation is produced in linear systems, the original two component model of Baym et al [1] posits a linear coupling between the crust and the superfluid interior. The equations of the linear two component model are

$$
\begin{aligned}
I_{\mathrm{c}} \dot{\Omega}_{\mathrm{c}}+I_{\mathrm{s}} \dot{\Omega}_{\mathrm{s}} & =N_{\mathrm{ext}}, \\
\dot{\Omega}_{\mathrm{s}}=-\frac{\Omega_{\mathrm{s}}-\Omega_{\mathrm{c}}}{\tau_{0}} & =-\frac{\omega}{\tau_{0}} .
\end{aligned}
$$

$I_{\mathrm{c}}$ and $I_{\mathrm{S}}$ denote the moments of inertia and $\Omega_{\mathrm{c}}$ and $\Omega_{\mathrm{s}}$ are the rotation rates of the crust and the superfluid, respectively. The rotational velocity lag between the two components is denoted by $\omega \equiv \Omega_{\mathrm{s}}-\Omega_{\mathrm{c}}$. The internal torque that the superfluid exerts on the crust is

$$
N_{\text {int }} \equiv-I_{\mathrm{s}} \dot{\Omega}_{\mathrm{s}}
$$


while $N_{\text {ext }}$ denotes the external torque on the neutron star. The crust (normal matter) rotation rate $\Omega_{\mathrm{c}}$ obeys the Navier Stokes Equation, but is already in rigid body rotation on superfluid timescales. The superfluid rotation rate $\Omega_{\mathrm{s}}$ does not obey the Navier Stokes Equation. Physically, the superfluid spins down by a flow of quantized vortices away from the rotation axis. Under many kinds of physical interactions between the vortices and normal matter, the vortex current, and the superfluid rate are linear in the angular velocity lag $\omega$.

Under a constant external torque $N_{\text {ext }}$, the system has a steady state,

$$
\begin{aligned}
\dot{\omega} & =0, \\
\dot{\Omega}_{\mathrm{s}} & =\dot{\Omega}_{\mathrm{c}}=\frac{N_{\mathrm{ext}}}{I} .
\end{aligned}
$$

The steady state value $\omega_{\infty}$ of the lag is determined by

$$
\frac{\omega_{\infty}}{\tau_{0}}=-\frac{N_{\mathrm{ext}}}{I} .
$$

The linear response to any glitch induced offset $\delta \omega(0)$ from steady state is exponential relaxation

$$
\dot{\Omega}_{\mathrm{c}}=\dot{\Omega}_{\mathrm{c}}(0)-\frac{I_{\mathrm{s}}}{I} \frac{\delta \omega(0)}{\tau} e^{-t / \tau} .
$$

Several components i of the exponential relaxation are indeed observed following pulsar glitches. An analysis of a postglitch relaxation yields $I_{\mathrm{s}, \mathrm{i}} / I \sim 10^{-3}-10^{-2}$ for each component of the exponential relaxation. This points at the crust superfluid. With the effective masses (entrainment) [4] taken into account, $I_{\mathrm{s}, \mathrm{i}} / I \lesssim 10^{-1}$, pointing to the crust + outer core [5]. The core superfluid is already coupled tightly to the crust; the core is effectively a part of the crust, dynamically, on glitch and postglitch relaxation timescales [6]. An analysis in terms of the two component model is enough: Since $I_{\mathrm{s}, \mathrm{i}} / I \ll 1$, the different superfluid components i with moments of inertia $I_{\mathrm{s}, \mathrm{i}}$ can be handled with the crust in separate two component models and then the response of the crust to each can be superposed.

\section{Vortex creep and the nonlinear two component model}

A superfluid component with vortex pinning can spin down by the thermally activated flow (creep) of vortices against pinning potentials. The vortex creep model gives the spindown rate

$$
\dot{\Omega}_{\mathrm{s}}=-\frac{4 \Omega_{\mathrm{s}} v_{0}}{r} \exp \left(-\frac{E_{\mathrm{p}}}{k T}\right) \sinh \left(\frac{\omega}{\varpi}\right) \equiv-f(\omega),
$$

where

$$
\varpi \equiv \frac{k T}{E_{\mathrm{p}}} \omega_{\mathrm{cr}},
$$

to replace (2). Here, $E_{\mathrm{p}}$ is the pinning energy and $T$ is the temperature. The distance $r$ of the vortex lines from the rotation axis is approximately equal to the neutron star radius $R$ in the crust superfluid where creep takes place against the pinning sites in the crust lattice. The microscopic vortex velocity $v_{0}$ around pinning centres is $\approx 10^{7} \mathrm{~cm} / \mathrm{s}[7]$. The two component model is now nonlinear in the lag $\omega$.

The values of the parameters, in particular of $E_{\mathrm{p}} / k T$, can be such that (7) requires $\sinh (\omega / \varpi) \gg 1$, so that $\sinh (\omega / \varpi) \simeq(1 / 2) \exp (\omega / \varpi)$. Equations $(1)$ and $(7)$ then yield

$$
\dot{\Omega}_{\mathrm{c}}=\frac{N_{\text {ext }}}{I_{\mathrm{c}}}+\frac{I_{\mathrm{s}}}{I_{\mathrm{c}}} \frac{\varpi}{2 \tau_{\mathrm{l}}} \exp (\omega / \varpi),
$$


where

$$
\tau_{1} \equiv \frac{k T}{E_{\mathrm{p}}} \frac{R \omega_{\mathrm{cr}}}{4 \Omega_{\mathrm{s}} v_{0}} \exp \left(\frac{E_{\mathrm{p}}}{k T}\right) .
$$

For nonlinear coupling, the steady state value of the lag, $\omega_{\infty}$, is given by (4) and (7).

The solution for the crust spindown rate under a constant external torque $N_{\text {ext }}=I \dot{\Omega}_{\infty}$ is

$$
\dot{\Omega}_{\mathrm{c}}(t)=\frac{I}{I_{\mathrm{c}}} \dot{\Omega}_{\infty}-\frac{I_{\mathrm{s}}}{I_{\mathrm{c}}} \dot{\Omega}_{\infty}\left[1+\left[\exp \left(\frac{t_{0}}{\tau_{\mathrm{nl}}}\right)-1\right] \exp \left(-\frac{t}{\tau_{\mathrm{nl}}} \frac{I}{I_{\mathrm{c}}}\right)\right]^{-1}
$$

with a nonlinear creep relaxation time

$$
\tau_{\mathrm{nl}} \equiv \frac{k T}{E_{\mathrm{p}}} \frac{I \omega_{\mathrm{cr}}}{N_{\mathrm{ext}}}=\frac{k T}{E_{\mathrm{p}}} \frac{\omega_{\mathrm{cr}}}{|\dot{\Omega}|_{\infty}}
$$

and recoupling (waiting) timescale

$$
t_{0} \equiv \frac{I \delta \omega}{N_{\mathrm{ext}}}=\frac{\delta \omega}{|\dot{\Omega}|_{\infty}}
$$

Nonlinear creep regions are responsible for glitches through vortex unpinning avalanches leading to a decrease $\delta \Omega_{\mathrm{s}}(r)$ in those superfluid regions through which the unpinned vortices move rapidly during the glitch. Angular momentum conservation leads to $\delta \Omega_{\mathrm{s}}=\left(I_{\mathrm{c}} / I_{\mathrm{s}}\right) \Delta \Omega_{\mathrm{c}} \gg \Delta \Omega_{\mathrm{c}}$, and $\delta \omega \cong \delta \Omega_{\mathrm{s}}$. Because of the very sensitive dependence of the creep rate on $\delta \omega$ this glitch induced offset from the steady state lag $\omega_{\infty}$ will stop creep altogether in the superfluid regions effected. As the same external torque is now acting on less moment of inertia, the observed spindown rate of the crust will suffer a glitch associated jump

$$
\frac{\Delta \dot{\Omega}_{\mathrm{c}}}{\dot{\Omega}_{\mathrm{c}}} \cong \frac{I_{\mathrm{s}}}{I_{\mathrm{c}}}
$$

Creep restarts after a waiting time $t_{0} \cong \delta \Omega_{\mathrm{s}} /|\dot{\Omega}|_{\infty}$. This "Fermi function" response was predicted as a standard signature of the vortex creep response to a glitch involving vortex unpinning (see figure 4 in [2]) and later observed in the Vela pulsar [8]. The common, almost ubiquitous form of nonlinear response is the constant second derivative $\ddot{\Omega}_{\mathrm{c}}$ interglitch timing behaviour observed in the Vela and other pulsars $[9,10]$. This is the response to a uniform density of vortices unpinned at the glitch leading to a range of waiting times throughout the superfluid regions. Such power law behaviour is characteristic of nonlinear dynamics.

Two component models with linear or nonlinear internal torques have so far been applied to post-glitch or inter-glitch timing behaviour of radio pulsars [9,11-14], where the external torque, with a secular (characteristic) timescale $\tau_{\mathrm{c}} \equiv \Omega_{\mathrm{c}} / 2\left|\dot{\Omega}_{\mathrm{c}}\right| \sim 10^{3}-10^{6}$ yr is constant for timescales of observed postglitch relaxation.

While constant secular external torques are characteristic for radio pulsars, neutron stars in $\mathrm{X}$-ray binaries, magnetars and transients exhibit strong variations in the observed spin-down or spin-up rates. This indicates variable external torques, including strong torque noise.

\section{Linear two-component model with a time dependent external torque}

The two component model postglitch response with a linear internal torque and a time varying external torque is described by

$$
\dot{\omega}=-\frac{\omega}{\tau}-\frac{N_{\mathrm{ext}}(t)}{I_{\mathrm{c}}} .
$$


The relaxation time $\tau$ is determined by the physics of the internal torque. The solution is

$$
\begin{aligned}
\omega(t) & =e^{-t / \tau}\left[\omega(0)-\frac{1}{I_{\mathrm{c}}} \int_{0}^{t} e^{t^{\prime} / \tau} N_{\mathrm{ext}}\left(t^{\prime}\right) \mathrm{d} t^{\prime}\right] \\
\dot{\Omega}_{\mathrm{c}}(t) & =\frac{N_{\mathrm{ext}}(t)}{I_{\mathrm{c}}}+\frac{I_{\mathrm{s}}}{I} \frac{e^{(-t / \tau)}}{\tau}\left[\omega(0)-\frac{1}{I_{\mathrm{c}}} \int_{0}^{t} e^{\left(t^{\prime} / \tau\right)} N_{\mathrm{ext}}\left(t^{\prime}\right) \mathrm{d} t^{\prime}\right] .
\end{aligned}
$$

\section{Vortex Creep Model with a Time Dependent External Torque}

For vortex creep under a time dependent external torque (1) and (7) lead to

$$
\dot{\omega}=-\frac{I \varpi}{2 I_{\mathrm{c}} \tau_{\mathrm{l}}} e^{\omega / \varpi}-\frac{N_{\mathrm{ext}}(t)}{I_{\mathrm{c}}} .
$$

In terms of $y \equiv \exp (-\omega / \varpi)$, equation (17) becomes

$$
\frac{\mathrm{d} y}{\mathrm{~d} t}-\frac{N_{\mathrm{ext}}(t)}{I_{\mathrm{c}} \varpi} y-\frac{I}{2 I_{\mathrm{c}} \tau_{\mathrm{l}}}=0 .
$$

This has an integration factor $\exp \left(-\frac{X(t)}{I_{\mathrm{c}} \varpi}\right)$ where

$$
X(t)=\int_{0}^{t} N_{\text {ext }}\left(t^{\prime}\right) \mathrm{d} t^{\prime}
$$

The solution for the angular velocity lag $\omega$ exhibits an exponential dependence on the postglitch lag $\omega(0)$ and on $X(t)$, the cumulative angular momentum transfer by the external torque:

$$
\begin{aligned}
e^{-(\omega / \varpi)} & =e^{-\omega(0) / \varpi} \exp \left(\frac{X(t)}{I_{\mathrm{c} \varpi}}\right) \\
& +\exp \left(\frac{X(t)}{I_{\mathrm{c} \varpi}}\right) \int_{0}^{t} \frac{I}{2 I_{\mathrm{c}} \tau_{\mathrm{l}}} \exp \left(-\frac{X\left(t^{\prime}\right)}{I_{\mathrm{c}} \varpi}\right) \mathrm{d} t^{\prime}
\end{aligned}
$$

From equations (1), (17), and (20) the response of nonlinear creep to a glitch in the presence of a time dependent external torque is obtained,

$$
\begin{aligned}
\dot{\Omega}_{\mathrm{c}}(t) & =\frac{N_{\mathrm{ext}}(t)}{I_{\mathrm{c}}} \\
& +\frac{I_{\mathrm{s}}}{I_{\mathrm{c}}} \frac{\varpi}{2 \tau_{\mathrm{l}}}\left[\exp \left(\frac{\frac{X(t)}{I_{\mathrm{c}}}-\omega(0)}{\varpi}\right)+\exp \left(\frac{X(t)}{I_{\mathrm{c}} \varpi}\right) \int_{0}^{t} \frac{I}{2 I_{\mathrm{c}} \tau_{\mathrm{l}}} \exp \left(-\frac{X\left(t^{\prime}\right)}{I_{\mathrm{c}} \varpi}\right) \mathrm{d} t^{\prime}\right]^{-1} .
\end{aligned}
$$

The nonlinear creep timescale and the waiting time are now time dependent, involving the running time average $\left\langle N_{\text {ext }}(t)\right\rangle \equiv X(t) / t$ of the external torque:

$$
\begin{aligned}
\tau_{\mathrm{nl}}(t) & \equiv \frac{k T}{E_{\mathrm{p}}} \frac{I \omega_{\mathrm{cr}}}{\left\langle N_{\mathrm{ext}}(t)\right\rangle}, \\
t_{0}(t) & \equiv \frac{I \delta \omega(0)}{\left\langle N_{\mathrm{ext}}(t)\right\rangle} .
\end{aligned}
$$




\section{Applications}

Equations (16) and (21) show that the observed $\dot{\Omega}_{\mathrm{c}}(t)$ gives $N_{\text {ext }}(t) / I_{\mathrm{c}}$ to lowest order in $I_{\mathrm{S}} / I_{\mathrm{c}} \lesssim 0.1$. The residuals after a first fit to the $\dot{\Omega}_{\mathrm{c}}(t)$ time series will be $I_{\mathrm{S}} / I_{\mathrm{c}}$ times a certain convolution of the external torque with the internal torque, as defined in (16) or (21), allowing for a consistency check of the model.

Let us consider three kinds of time dependent external torques; (i) an exponentially decaying external torque, (ii) power law time dependence and (iii) timing noise.

(i) Taking an exponentially decaying torque with time scale $\tau_{\mathrm{d}}$ added to the preglitch external torque $N_{0}$, we have

$$
N_{\text {ext }}(t)=N_{0}+\delta N e^{-t / \tau_{\mathrm{d}}}=I \dot{\Omega}_{\infty}+\delta N e^{-t / \tau_{\mathrm{d}}} .
$$

For linear internal torques, (16) gives

$$
\begin{aligned}
\dot{\Omega}_{\mathrm{c}}(t) & =\dot{\Omega}_{\infty}+\frac{\delta N}{I_{\mathrm{c}}} e^{-t / \tau_{\mathrm{d}}}\left[1-\frac{I_{\mathrm{s}}}{I} \frac{\tau_{\mathrm{d}}}{\tau_{\mathrm{d}}-\tau}\right] \\
& +\frac{I_{\mathrm{s}}}{I} e^{-t / \tau}\left(\frac{\omega(0)}{\tau}+\frac{I}{I_{\mathrm{c}}} \dot{\Omega}_{\infty}+\frac{\delta N}{I_{\mathrm{c}}} \frac{\tau_{\mathrm{d}}}{\tau_{\mathrm{d}}-\tau}\right) .
\end{aligned}
$$

For vortex creep, the solution is obtained by substituting in (21) the integrated angular momentum transfer

$$
X(t)=N_{0} t+\delta N \tau_{\mathrm{d}}\left[1-e^{-t / \tau_{\mathrm{d}}}\right] .
$$

(ii) For a power law torque with the index $\alpha$ added to the preglitch torque we have

$$
N_{\text {ext }}(t)=N_{0}+\frac{\delta N t_{0}^{\alpha}}{\left(t+t_{0}\right)^{\alpha}}=I \dot{\Omega}_{\infty}+\frac{\delta N t_{0}^{\alpha}}{\left(t+t_{0}\right)^{\alpha}}
$$

Then (16) gives

$$
\begin{aligned}
\dot{\Omega}_{\mathrm{c}}(t) & =\dot{\Omega}_{\infty}+\frac{\delta N}{I_{\mathrm{c}}} \frac{t_{0}^{\alpha}}{\left(t+t_{0}\right)^{\alpha}}\left[1-\frac{I_{\mathrm{s}}}{I} \frac{\left(t+t_{0}\right)}{(1-\alpha) \tau}\right] \\
& +\frac{I_{\mathrm{s}}}{I} e^{-t / \tau}\left(\frac{\omega(0)}{\tau}+\frac{I}{I_{\mathrm{c}}} \dot{\Omega}_{\infty}+\frac{\delta N}{I_{\mathrm{c}}} \frac{t_{0}}{(1-\alpha) \tau}+\frac{I_{\mathrm{s}}}{I} \frac{\delta N}{I_{\mathrm{c}}} \frac{t_{0}^{\alpha}}{(1-\alpha) \tau^{2}} \int_{0}^{t} \frac{e^{t^{\prime} / \tau} \mathrm{d} t^{\prime}}{\left(t+t_{0}\right)^{\alpha-1}}\right),
\end{aligned}
$$

for linear internal torques.

The integrated angular momentum transfer $X(t)$ is

$$
X(t)=N_{0} t+\frac{\delta N t_{0}}{\alpha-1}\left[1-\frac{t_{0}^{\alpha-1}}{\left(t+t_{0}\right)^{\alpha-1}}\right]
$$

defining the response with nonlinear internal torques through Equation (21).

(iii) Take white torque noise:

$$
N_{\text {ext }}=\sum_{\mathrm{i}} \alpha_{\mathrm{i}} \delta\left(t-t_{\mathrm{i}}\right)
$$

where $\alpha_{\mathrm{i}}$ are amplitudes of torque variations. For linear coupling equations (16) and (30) lead to the power spectrum

$$
\begin{aligned}
P(f) & =\frac{1}{\sqrt{2 \pi}}\left[\frac{2\left\langle\alpha^{2}\right\rangle}{I_{\mathrm{c}}^{2}}+\left(\frac{I_{\mathrm{s}} / I}{1+(2 \pi \tau f)^{2}}\right)\left(\frac{\langle\alpha\rangle\langle\omega\rangle}{I_{\mathrm{c}}}-\frac{\left\langle\alpha^{2}\right\rangle}{I_{\mathrm{c}}^{2}}\right)\right. \\
& \left.+\left(\frac{\left(I_{\mathrm{s}} / I\right)^{2}}{1+(2 \pi \tau f)^{2}}\right)\left(\frac{2\left\langle\alpha^{2}\right\rangle}{I_{\mathrm{c}}^{2}}+2\left\langle\omega^{2}\right\rangle-\frac{\langle\alpha\rangle\langle\omega\rangle}{I_{\mathrm{c}}}\right)\right],
\end{aligned}
$$


where $\langle\alpha\rangle$ is the mean external torque variation amplitude and $\langle\omega\rangle$ denotes the mean value of the angular velocity lag. This result was obtained earlier for the linear two component model [15-17]. To lowest order we find the model power spectrum, a constant $P(f)$ for white noise. To order $I_{\mathrm{s}} / I$ we find the power spectrum of the integrated process, which is flat at low $f$ and a random walk spectrum, $P(f) \propto f^{-2}$, at high frequencies $f \gg \tau^{-1}$. The first fit will give the strength of the noise process, the term proportional to $\left\langle\alpha^{2}\right\rangle$. The residuals to order $I_{\mathrm{S}} / I$ will constrain $\langle\alpha\rangle\langle\omega\rangle$ and $\left\langle\omega^{2}\right\rangle$. The term proportional to $\left(I_{\mathrm{s}} / I\right)^{2}$ can be neglected to a good approximation. Noise processes other than white noise can be handled similarly for the linear two component model, by inserting the noise model in (16) and calculating the power spectrum. For the nonlinear two component model, equation (21) does not lead to explicit expressions for any time dependent external torque, but a well defined algorithm can be constructed to obtain the power spectrum, as for the times series in cases (i) and (ii).

\section{Acknowledgments}

This work was supported by the Scientific and Technological Research Council of Turkey (TÜBİTAK) under the grant 113F354. M.A.A. is a member of the Science Academy (Bilim Akademisi), Turkey.

\section{References}

[1] Baym G, Pethick C J, Pines D and Ruderman M 1969 Nature 224872

[2] Alpar M A, Anderson P W, Pines D and Shaham J 1984 ApJ 276325

[3] Gügercinoğlu E and Alpar, M A 2017 MNRAS 4714827

[4] Chamel N 2012 Phys. Rev. C 85035801

[5] Gügercinoğlu E and Alpar, M A 2014 ApJ 788 L11

[6] Alpar M A, Langer S A and Sauls J A 1984b ApJ 282533

[7] Gügercinoğlu E and Alpar M A 2016 MNRAS 4621453

[8] Buchner S and Flanagan C 2008 American Institute of Physics Conference Series vol 983, ed C Bassa, Z Wang, A Cumming and V Kaspi p 145

[9] Akbal O, Alpar MA, Buchner S and Pines D 2017 MNRAS 4694183

[10] Yu M et al 2013, MNRAS 429688

[11] Alpar M A, Chau H F, Cheng K S and Pines D 1993 ApJ 409345

[12] Alpar M A, Chau H F, Cheng K S and Pines D 1996 ApJ 459706

[13] Akbal O, Gügercinoğlu E, Şaşmaz Muş S and Alpar M A 2015 MNRAS 449933

[14] Gügercinoğlu E 2017, MNRAS 4692313

[15] Lamb F K, Pines D and Shaham J 1978a ApJ 224969

[16] Lamb F K, Pines D and Shaham J 1978b ApJ 225582

[17] Baykal A, Alpar M A and Kızıloğlu Ü 1991, A\&SA 252664 